\title{
Hemolytic Uremic Syndrome: New Developments in Pathogenesis and Treatment
}

\author{
Olivia Boyer and Patrick Niaudet \\ Service de Néphrologie Pédiatrique, Hôpital Necker-Enfants Malades, 149 rue de Sèvres, 75015 Paris, France \\ Correspondence should be addressed to Patrick Niaudet, pniaudet@gmail.com
}

Received 30 April 2011; Accepted 14 June 2011

Academic Editor: Franz Schaefer

Copyright ( $) 2011$ O. Boyer and P. Niaudet. This is an open access article distributed under the Creative Commons Attribution License, which permits unrestricted use, distribution, and reproduction in any medium, provided the original work is properly cited.

\begin{abstract}
Hemolytic uremic syndrome is defined by the characteristic triad of microangiopathic hemolytic anemia, thrombocytopenia, and acute renal failure. In children, most cases of HUS are caused by Shiga-toxin-producing bacteria, especially Escherichia coli O157:H7. Common vehicles of transmission include ground beef, unpasteurized milk, and municipal or swimming water. Shigatoxin-associated HUS is a main cause of acute renal failure in young children. Management remains supportive as there is at present no specific therapy to ameliorate the prognosis. Immediate outcome is most often favourable but long-term renal sequelae are frequent due to nephron loss. Atypical HUS represents $5 \%$ of cases. In the past 15 years, mutations in complement regulators of the alternative pathway have been identified in almost $60 \%$ of cases, leading to excessive complement activation. The disease has a relapsing course and more than half of the patients either die or progress to end-stage renal failure. Recurrence after renal transplantation is frequent.
\end{abstract}

\section{Introduction}

The hemolytic-uremic syndrome (HUS) is defined by the association of hemolytic anemia (low haptoglobin levels, high lactate deshydrogenase levels, and schistocytes), thrombocytopenia, and acute renal failure [1]. It is a common cause of acute renal failure in patients younger than three years who require acute dialysis. The renal histological lesion is glomerular thrombotic microangiopathy and in more severe cases, arteriolar thrombotic microangiopathy. Increased platelet aggregation and thrombi affect the microcirculation. Thrombotic microangiopathy may affect other organ systems, including the central nervous system [2].

HUS can be divided into two forms, typical and atypical HUS. Typical $\mathrm{D}^{+}$or shiga-toxin associated HUS is the most common form of HUS in children accounting for 90 percent of all cases. It usually occurs after a prodromal episode of diarrhea that is frequently bloody. In the majority of cases, HUS is associated Shiga toxin-producing enterohemorrhagic Escherichia coli (EHEC) or Shigella [3-7] and generally carries a good prognosis [6]. Atypical HUS (aHUS) is a heterogeneous disorder which is responsible for only 10 percent of cases in children $[8,9]$. It is also referred to as nondiarrhea-associated HUS, D-HUS, or sporadic HUS. In addition to the usual absence of a diarrheal prodrome, children with aHUS may have an insidious onset, a relapsing course, or a progressive course leading to severe renal dysfunction $[1,8,10]$. Blood pressure is often markedly increased. There is a high risk of recurrence after renal transplantation.

\section{Shiga-Toxin-(Stx-) Associated HUS}

In the majority of cases, Stx-HUS is triggered by strains of EHEC that produce a Shiga toxin $[3,7,11]$, but Shigella dysenteriae can also be involved $[4,12]$. It usually occurs after a prodromal episode of diarrhea that is frequently bloody in nature. Five to 10 days after onset, HUS develops abruptly $[13,14]$. Cases of HUS in children due to Shiga toxin-producing E. coli infections other than colitis (e.g., urinary tract infections) can occur [15]. Although these cases are not associated with diarrhea, the clinical course and pathophysiology are the same as the $\mathrm{D}+$ HUS. 
2.1. Epidemiology. Stx-HUS principally affects young children. The annual incidence of the disease in North America and Western Europe is about 2 to 3 per 100,000 children less than five years of age $[6,12]$. Stx-HUS occurs most commonly in the summer months [11] and is more frequent in rural versus urban populations [7]. Most cases are sporadic, but outbreaks are often reported due to a common contaminated food or water source. The natural reservoir of EHEC is the digestive tract of healthy cattles Infection in humans occurs following ingestion of contaminated undercooked meat, unpasteurized milk or milk products, water, fruits, or vegetables [16]. Secondary human-tohuman transmission is also a major risk factor for HUS $[14,17]$ and may be a concern in day-care centers or in sibships.

EHEC causes at least 70 percent of cases of postdiarrheal HUS in the United States, 80 percent of which are caused by E. coli O157:H7, but non-O157 strains are also involved. Although laboratory diagnosis of EHEC infection [18] can be made by stool culture, the bacteria are only present in the stools for a few days and, even if present, may not be detected by culture from stool samples. The rate of stool isolation is substantially higher in the first six days after onset of diarrhea. Direct detection of shigatoxin in stool is another diagnostic tool. Only $10-15 \%$ of children with E. coli O157 colitis eventually develop HUS [19] suggesting that, in addition to pathogen factors, host factors also contribute to its development. Shigella dysenteriae type 1-associated HUS occurs in India, Bangladesh, and southern Africa. Although the pathogenesis of disease is similar to that of HUS induced by $\mathrm{O} 157 \mathrm{E}$. coli infection, the disease is usually more severe with an acute mortality rate of 15 percent and over 40 percent of patients developing chronic renal failure [12].

E. coli infection has become a major health care concern. Prevention recommendation should be provided to patients and their parents including meticulous hygiene when cooking and changing diapers, avoidance of undercooked meat and unpasteurized milk products in young children. Cattle vaccination could also help reducing the reservoir of E. coli. Promising results have been obtained in reducing the fecal shedding of E. coli O157:H7 and hide contamination following cattle vaccination [20-22]. But larger epidemiological studies are needed to assess a potential beneficial effect of cattle vaccination for food and environmental safety.

2.2. Pathophysiology. Shiga toxin-(Stx-) mediated injury to vascular endothelial cells in the kidney, brain, and other organs underlies the pathogenesis of HUS caused by EHEC. These potent cytotoxins are released in the gut by bacteria, enter the blood stream, and cause endothelial injury through the binding to the globotriaosylceramide (Gb3) receptor on the plasma membrane of target cells [23]. Gb3 is a sphingolipid receptor expressed on endothelial cells, podocytes, and proximal tubular cells in human. Stx binding to Gb3 leads to Stx internalization by receptormediated endocytosis and its retrograde transport to the endoplasmic reticulum. This triggers a cascade of signalling events, involving NF- $\kappa \mathrm{B}$ activation, which induces apoptosis and the binding of leukocytes to endothelial cells [24].
Stx-activated endothelial cells become thrombogenic by a complex mechanism not yet fully unravelled [24]. It has been demonstrated that Stxs modulate the expression of endothelial cell adhesive molecules such as $\beta 3$-integrin subunit, vitronectin receptor, PECAM-1, and P-selectin on the microvasculature [25] and induce the release of cytokines and chemokines by endothelial cells, implicated in platelet activation [26]. Moreover, upon Stx activation, endothelial Von Willebrandt factor has been shown to undergo conformational changes thereby mediating platelet adhesion on activated endothelial cells [25]. Additionally, Stxs stimulate in vitro the release of unusually large Von Willebrandt factor strings and impair their cleavage by the metalloprotease ADAMTS-13 [27]. Altogether, these endothelial cell dysfunctions associated to platelet activation may promote platelet adhesion on glomerular endothelial cells, thrombi formation, and glomerular occlusion in StxHUS. An in vitro study showed that in addition to directly damaging the kidney, Stxs also activate complement and delay the function of its inhibitor, factor $\mathrm{H}$, on the cell surface, leading to indirect kidney damage [28].

Stxs include two major antigenic forms (Stx1 and Stx2) with different subtypes which differ in potency. Stx2a and Stx2d have been reported as the most potent [29]. Stx typespecific mechanisms of endothelial cell injury have been suggested in cellular models [30]. Bauwens et al. demonstrated that Stx1 causes both necrosis and apoptosis, whereas Stx2 induces almost exclusively apoptosis in human brain microvascular endothelial cell lines and inmacrovascular endothelial cells [30]. Free toxins have never been detected in the blood of HUS patients, but they have been found on the surface of polymorphonuclear leukocytes [31]. Purified Stxs alone are capable of producing HUS with glomerular thrombotic microangiopathy in large animal models such as baboons [32]. Conversely, rodents develop tubular lesions but no glomerular damage after Stx injection. This may be related to Gb3 expression differences among species. Indeed, Gb3 expressed on tubular cells is absent from glomerular endothelial cells and podocytes in mice [23]. Similarly, cattle which are the main vectors of EHEC lack Gb3 expression in their glomeruli. This may partly explain why they do not develop HUS [33]. This led to the hypothesis that different patterns of Gb3 expression with age could explain the predominance of HUS among young children. However, it was shown that Gb3 expression in the kidney and Stx1 binding to kidney structures does not vary with age [34]. Factors other than basal renal Gb3 expression, yet to be identified, account for the age-related incidence of Stx-HUS in EHEC infection. The increased frequency of antibodies to Stx 1 and Stx 2 suggests that preexisting immunity may play a role in age-specific host resistance to HUS [35].

2.3. Clinical Manifestations. Stx-producing E. coli causes a spectrum of outcomes ranging from asymptomatic carriage to diarrhea that may be uncomplicated or bloody to HUS. The diarrhea and associated gastrointestinal complaints in the prodromal phase of Stx-HUS may mimic those of ulcerative colitis, other enteric infections, and appendicitis. HUS is defined by the sudden onset of microangiopathic 
hemolytic anemia with fragmented erythrocytes named schistocytes and negative Coombs' tests, thrombocytopenia, and acute kidney injury [11]. Despite the thrombocytopenia, there is usually no purpura or active bleeding. Whereas the degree of anemia or thrombocytopenia is unrelated to the severity of renal dysfunction, an increased white blood cell count is associated with a worse prognosis [36]. The hematologic manifestations of Stx HUS completely resolve usually within one to two weeks.

The renal involvement ranges from hematuria usually microscopic and proteinuria, to severe renal failure and oliguria that occur in one-half of cases. Hypertension is common. Dialysis is instituted to correct metabolic abnormalities when required. The short-term renal prognosis is generally favourable. However, the risk of renal failure 20 years after the recovery of Stx-HUS is not negligible, and renal histology showing a glomerular microangiopathy affecting $>50$ percent of glomeruli, arterial microangiopathy, and/or cortical necrosis is the best indicator of long-term prognosis $[37,38]$.

Stx-HUS commonly affects other organ systems [36]. Central nervous system involvement occurring in 20-50 percent of children with Stx-HUS is the most threatening complication associated with increased morbidity and mortality $[2,39,40]$. Patients may present with seizures, coma, stroke, hemiparesis, facial palsy, pyramidal or extrapyramidal syndromes, dysphasia, diplopia and cortical blindness [39]. Brain MRI typically reveals bilateral hypersignal on T2weighted and hyposignal on T1-weighted images of basal ganglia, thalami, and brainstem sometimes extending to the surrounding white matter [41]. Additionally, MRI may display images of high blood pressure complications such as reversible posterior leukoencephalopathy syndrome and cerebral hemorrhage. In a large French multicenter series of 52 children with Stx-HUS and neurological involvement, nine patients died, 17 had mild to severe sequelae, and 26 fully recovered [2].

The whole digestive tract can also be involved. The more serious manifestations include severe hemorrhagic colitis, bowel necrosis and perforation, rectal prolapse, peritonitis, and intussusception [42]. Cardiac ischemia and dysfunction may occur. Transient and rarely permanent diabetes mellitus may occur [42]. Hepatomegaly and/or increased serum transaminases are frequently observed. The mortality rate has dropped to about $5 \%$, usually due to neurologic or cardiac involvement [43]. Persistent oliguria ( $>5$ days of anuria and $>10$ days of oliguria), dehydration, elevated white blood cells $>20,000$ per $\mathrm{mm}^{3}$, and hematocrit $>23$ percent are risk factors for death [43] and long-term complications from HUS [44].

2.4. Therapy. Once a patient is infected with EHEC, attempts to prevent progression from the bloody diarrheal phase to the postdiarrheal phase of HUS have been unsuccessful. Antimotility drugs (such as anticholinergic agents and narcotics) do not reduce the progression to HUS due to EHEC infections, but in fact appear to increase the risk of subsequent development of HUS. In patients with EHEC, both retrospective and prospective observational studies report an increased risk of HUS with the administration of antibiotics during the bloody diarrheal phase [45]. In one retrospective review of 278 children with HUS, the use of antimotility drugs (anticholinergic agents and narcotics) was associated with an increased risk of subsequent development of HUS (OR 2.9, 95\% CI 1.2 to 7.5) [46]. In another retrospective study of 91 patients, antimotility drugs increased the risk of central nervous system dysfunction (OR 8.5, 95\% CI 1.7 to 42.8) [40]. Based upon these data, antibiotics and antimotility drugs should not be given to a child with confirmed or suspected E. coli O157:H7 infection. Increased volume expansion with intravenous isotonic saline during the diarrheal phase has been reported to attenuate but not prevent renal injury $[6,47]$.

Treatment of Stx-HUS includes supportive measures such as management of anemia, significant clinical bleeding, fluid and electrolyte disturbances, hypertension, and other extrarenal complications, together with renal replacement therapy required in $30-60 \%$ of cases [48]. Additionally, multiple modalities and/or agents have been utilized that are directed against the underlying or presumed pathogenic mechanisms of Stx-HUS. These include antithrombotic agents, plasma exchange and/or plasma infusion, tissuetype plasminogen activator, and oral Shiga toxin-binding agent. Although none of these agents have been shown to be efficacious, there may be a role for plasma exchange in patients with severe CNS involvement. The remaining agents which have not proven efficient are not recommended. Stxreceptor (Gb3) mimics and Stx-neutralizing (monoclonal) antibodies $(\mathrm{STm} A \mathrm{~b})$ may be more promising. Although the first-generation Gb3-analog failed to ameliorate the disease course in a randomized placebo-controlled trial [49], advanced Stx receptor analogs and polymers, and peptidebased intracellular toxin inhibitors have been developed and could have more beneficial effects in the future [5052]. STmAb injection $24 \mathrm{~h}$ after oro-gastric inoculation of Stx2-producing bacteria prolonged the survival of toxinchallenged mice [53]. This provided the rationale for the use of STmAb products for Phase 1 studies in patients in which a satisfactory tolerability was demonstrated [54]. Randomized controlled trials are needed to confirm the potential role of STmAb in patients with Stx-HUS. Eculizumab looks promising in severe cases with neurological involvement refractory to plasma therapy [55]. Finally, treatments targeting downstream events in the inflammatory or proapoptotic cascade induced by Stx could be a good option, yet to be developed.

\section{Atypical HUS}

An increasing number of genetic causes of aHUS have been described [1]. These include aHUS associated with mutation of genes encoding for regulators of the alternative complement pathway, Von Willebrand factor cleaving protease (ADAMTS13) deficiency, and intracellular defects of vitamin B12 metabolism. There are also cases of unknown etiology that are associated with an autosomal recessive and autosomal dominant inheritance. 
3.1. Atypical HUS and Defective Complement Regulation. Mutations in the genes encoding complement proteins including $\mathrm{C} 3$, factors $\mathrm{H}, \mathrm{B}$, and I, and membrane cofactor protein (MCP) are associated with atypical HUS [56, 57]. It is estimated that approximately 60 percent of cases of aHUS result from mutations in these genes [56]. These mutations result in dysregulation of the complement system that leads to excessive complement activation and in endothelial damage.

The gene that is affected determines the clinical presentation and outcome $[58,59]$. For example, patients with mutations of the gene for factor $\mathrm{H}(C F H)$ often progress to ESRD within the first year of presentation. By contrast, only few patients with MCP mutations progress to ESRD, although relapse is common [59]. Interestingly, only $50 \%$ of individuals with $C F H$ or $C F I$ or MCP mutation eventually develop aHUS, which mean that additional risk factors are involved in the development of the disease. Combined mutations of the genes encoding complement proteins are observed in $5 \%$ of patients. The association of $C F H$ and MCP haplotypes with aHUS has been reported [60].

3.1.1. Factor $H$ Mutations. The association between aHUS and mutations in $\mathrm{CFH}$ was first described by Warwicker et al. [61]. It was subsequently found that it is the most frequent genetic abnormality in patients with aHUS, accounting for 25 to $30 \%$ of cases [62-65]. Most mutations are heterozygous. Factor $\mathrm{H}$, a serum complement regulatory protease, acts as a cofactor for factor I-mediated inactivation of C3b, competes with factor B for C3b binding, and accelerates C3 convertase decay. More than 70 mutations have been reported. Most mutations affect the short consensus repeats 19 and 20 of the protein. Most of them are missense mutations, which do not affect the levels of factor $\mathrm{H}$ and C3 but affect the C-terminal region, which is important for binding to $\mathrm{C} 3 \mathrm{~b}$ and anionic surfaces $[66,67]$. Other mutations are located throughout the gene and are associated with low levels of factor $\mathrm{H}$ antigen. Interestingly, renal survival is higher in patients with low factor $\mathrm{H}$ levels compared with those with normal levels of factor $\mathrm{H}$. Patients with homozygous mutations have very low levels of factor $\mathrm{H}$ antigen, low $\mathrm{C} 3$, low factor $\mathrm{B}$, and low $\mathrm{CH} 50$. aHUS associated with factor $\mathrm{H}$ mutations may present during infancy or early childhood, or in adulthood $[62,63,68-$ 71 . The disease may be sporadic or clearly associated with a family history of disease. Hemolytic anemia is marked at disease onset and during relapses, with haptoglobin levels remaining low during the course of the disease. Hypertension is frequently severe. Progression to end-stage renal disease is often rapid, and there is a very high risk of recurrence after renal transplantation (75 to $90 \%)[72,73]$.

3.1.2. Mutations in MCP (Membrane Cofactor Protein). Mutations in the complement regulatory protein MCP are observed in 5 to $10 \%$ of aHUS $[58,74-76]$. MCP is the cofactor of factor I for the degradation of $\mathrm{C} 3 \mathrm{~b}$ and $\mathrm{C} 4 \mathrm{~b}$. The mutation results either in a reduction of cell surface levels of MCP (most common) or an impaired function of MCP [75]. The disease is characterized by onset in childhood, a favourable renal outcome in most patients, frequent relapses and a low rate of recurrence in the renal allograft.

3.1.3. Factor I Mutations. Factor I is a serine protease which cleaves $\mathrm{C} 3 \mathrm{~b}$ and $\mathrm{C} 4 \mathrm{~b}$ in the presence of factor $\mathrm{H}$ and MCP. The frequency of CFI mutations in patients with aHUS varies between 3 and 10\% [77]. Up to 15 different mutations have been identified [78]. Most patients have heterozygous mutations [79]. They result in either a quantitative defect or a qualitative defect of the protein. HUS often occur in early childhood, and progression to end-stage renal disease is observed in more than $50 \%$ of cases [80]. Other genetic anomalies are frequent and may explain a worse outcome in some patients. Recurrence occurs in 45 to $80 \%$ of patients, resulting in graft loss.

3.1.4. C3 Mutations. Heterozygous $\mathrm{C} 3$ mutations have been identified in patients with aHUS [81]. Fremeaux-Bacchi et al. reported 9 novel mutations in 14 patients with aHUS and persistently low serum C3 levels. Five of the seven C3 mutant proteins showed a reduced binding to MCP, possibly leading to a gain of function relative to complement activation.

The mean age at presentation was 6.5 years (range 8 months to 40 years). Seven of the 14 patients regained function after their initial presentation and four of these patients had recurrent disease. Among the 14 patients, there have been 12 renal transplantations, five of which have had recurrent disease.

3.1.5. Factor B Mutations. Mutations of complement factor B that either enhance the formation or delay the inactivation of $\mathrm{C} 3 \mathrm{bBb}$ convertase have been reported in 1 to $3 \%$ of cases of aHUS [82, 83]. Progression to end-stage renal disease occurs in $70 \%$ of patients and all four renal transplantation reported failed because of HUS recurrence.

3.1.6. Thrombomodulin Mutations. Thrombomodulin is a cofactor in the initiation of the protein $\mathrm{C}$ anticoagulant pathway. In addition, it was shown that thrombomodulin accelerates the inactivation of C3b by factor I. In a study of 152 patients with atypical HUS, seven patients had six different heterozygous mutations of the THBD gene, which encodes thrombomodulin [84]. In vitro studies demonstrated that these mutations resulted in a decreased capacity to inactivate C3b, which may lead to excessive complement activation.

3.1.7. Antifactor H Autoantibody-Associated aHUS. The presence of autoantibodies against factor $\mathrm{H}$ is observed in 6 to $10 \%$ of cases, mainly in children, between 9 and 12 years. These antibodies interfere with the binding of factor $\mathrm{H}$ to the $\mathrm{C} 3$ convertase and are associated with a defective factor $\mathrm{H}$-dependent cell protection $[85,86]$. Most patients have a homozygous deletion of CFHR1 and CFHR3 genes [87-89]. The clinical course is characterized by a high frequency of relapses. Progression to end-stage renal disease is observed in 20 to $35 \%$ of cases [90]. Plasma exchange plus immunosuppressive therapy have been successful measures 
in some patients with factor $\mathrm{H}$ antibodies [91]. Recurrence is observed when the autoantibodies are still present at time of transplantation [92].

3.1.8. Treatment. Intensive plasma exchange (40 to $60 \mathrm{~mL} / \mathrm{kg}$ ) is the first-line therapy for patients during the acute episode of aHUS. The response to plasma treatment appears to vary depending upon the affected complement component [58, 93]. In patients with either factor $\mathrm{H}$ or factor I deficiencies, about two-thirds of patients will remit with plasma therapy. In contrast, there has been no difference in remission rates with or without plasma therapy in patients with MCP deficiency. Patients with antifactor $\mathrm{H}$ autoantibodies should receive an immunosuppressive treatment in addition to plasma exchanges.

Eculizumab is a monoclonal antibody which binds to C5 and prevents the generation of C5a and the formation of the membrane attack complex. Eculizumab has been used in patients with aHUS on native kidney or recurrence of aHUS after transplantation with very encouraging results [94-97]. A prolonged therapy is more effective than a single dose. The delay between two injections should not exceed 14 days. The duration of therapy is still a matter of debate. A multicenter trial in children to establish the efficacy and safety of eculizumab in aHUS is ongoing.

Patients with mutations in genes for factor $\mathrm{H}$, factor I, or C3 who fail to respond to plasma therapy and/or have recurrent disease are likely to progress to ESRD [98]. It is unclear whether renal transplantation is an appropriate intervention in these patients, because recurrence of disease occurs in 50 percent of the transplanted kidney, and graft failure occurs in 90 percent of those with recurrent disease $[72,99]$. Genotyping for complement protein genes should be performed in patients who have atypical HUS and end-stage renal failure and are being considered for renal transplantation. Indeed, the risk of recurrence is dependent on the type of mutation; such genotyping is particularly important when living-related renal transplantation is being considered, so that cases in which de novo HUS occurs in the donor can be avoided. Most clinicians agree that living donor renal transplantation is contraindicated in patients with atypical HUS because of the high risk of disease recurrence after transplantation [1].

In 2007, a consensus conference recommended a combined liver-renal transplantation in aHUS cases in which renal transplantation is extremely unlikely to succeed [100, 101]. This would include patients with factor $\mathrm{H}$ or I deficiency who have lost an isolated kidney transplant due to disease recurrence or have a family member with the same gene mutation who lost a kidney transplant due to disease recurrence. The transplantation should be preceded by plasma exchange, and plasma should be infused intraoperatively and continued until liver function is adequate. Eculizumab is another option $[94,96]$.

\subsubsection{Atypical HUS and Von Willebrand Factor Cleaving} Protease Deficiency. Von Willebrand factor (VWF), a glycoprotein that carries factor VIII in the circulation, is required for platelet adhesion and platelet aggregation [102]. Large multimers of VWF are more effective than dimers for platelet adhesion and platelet aggregation. The large multimers do not normally circulate because they are cleaved by a specific protease, a metalloprotease synthesized by the liver. This protease is the thirteenth member of the ADAMTS family, or ADAMTS13.

Many cases of adult thrombotic thrombocytopenic purpura (TTP) are due to deficient activity of the VWF cleaving protease. Protease deficiency may be inherited by autosomalrecessive transmission or may be induced by an acquired autoantibody that inhibits protease activity $[103,104]$. A few cases of antibody-induced VWF cleaving protease deficiency have been reported in children aged 1 to 11 years who presented with symptoms of TTP.

Most children with VWF-cleaving protease deficiency related to mutations in the ADAMTS13 gene present at birth with hemolytic anemia and thrombocytopenia. Renal involvement often occurs later in life and has a progressive course. Onset of disease up to five years of age has also been reported. Many develop symptoms of CNS involvement.

3.1.10. Atipical HUS and Defects of Vitamin B12 Metabolism. Vitamin B12 or cobalamin is the coenzyme of methioninesynthase (or methyl-transferase), which transforms homocysteine in methionine. It is also the coenzyme of methylmalonyl-CoA mutase, which is involved in the conversion of methylmalonyl CoA to succinyl-CoA [105]. Children affected with cobalamin $\mathrm{C}$ mutations have a functional defect of both methylmalonyl CoA mutase and methioninesynthase. This defect results in methylmalonic acidemia with homocystinuria.

Approximately 25 percent of reported cases also have atypical HUS [105]. The first symptoms, which are nonspecific, occur in the neonatal period and consist of anorexia, vomiting, failure to thrive, hypotonia, seizures, and lethargy $[106,107]$. The first symptoms of HUS are observed between the end of the first month and three months of age with severe hemolytic anemia with schistocytes, which is accompanied by macrocytosis, frequent thrombocytopenia, hematuria, proteinuria, renal dysfunction of variable severity, and hypertension.

Amino acid and organic acid chromatography reveal a marked increase of homocysteine and a low level of methionine in the plasma, while urinary excretion of homocysteine and methylmalonic acid is very high. Therapy with hydroxycobalamin should be started as soon as possible [107].

3.2. Other Causes of Atypical HUS. HUS may occur during treatments with cytotoxic drugs, such as mitomycin C, bleomycin, or cisplatin $[108,109]$. HUS has been described in patients with HIV infection [110, 111]. HUS has been observed in patients with systemic lupus erythematosus [112] as well as in those with a catastrophic antiphospholipid syndrome.

3.3. HUS after Renal Transplantation. HUS may be observed following renal transplantation either as a recurrent or a de novo disease [113]. More than $50 \%$ of patients with aHUS 
have mutations in genes coding for regulatory factors of the complement system or antibodies against factor $\mathrm{H}$. The risk of recurrence depends on the genetic defect. When the risk of recurrence is high, as in patients with mutations in genes coding for circulating regulators of complement, isolated renal transplantation is contraindicated given the high risk of recurrence, although the use of eculizumab may allow such procedure.

Drug-induced HUS has been observed in transplant patients treated with either cyclosporine or tacrolimus [114, 115]. It usually occurs during the first few months posttransplantation, a time when high doses of calcineurin inhibitors are being administered.

\section{References}

[1] M. Noris and G. Remuzzi, "Hemolytic uremic syndrome," Journal of the American Society of Nephrology, vol. 16, no. 4, pp. 1035-1050, 2005.

[2] S. Nathanson, T. Kwon, M. Elmaleh et al., "Acute neurological involvement in diarrhea-associated hemolytic uremic syndrome," Clinical Journal of the American Society of Nephrology, vol. 5, no. 7, pp. 1218-1228, 2010.

[3] M. Fitzpatrick, "Haemolytic uraemic syndrome and E coli O157," British Medical Journal, vol. 318, no. 7185, pp. 684685, 1999.

[4] V. Houdouin, C. Doit, P. Mariani et al., "A pediatric cluster of Shigella dysenteriae serotype 1 diarrhea with hemolytic uremic syndrome in 2 families from France," Clinical Infectious Diseases, vol. 38, no. 9, pp. e96-e99, 2004.

[5] H. A. Repetto, "Epidemic hemolytic-uremic syndrome in children," Kidney International, vol. 52, no. 6, pp. 1708-1719, 1997.

[6] P. I. Tarr, C. A. Gordon, and W. L. Chandler, "Shigatoxin-producing Escherichia coli and haemolytic uraemic syndrome," The Lancet, vol. 365, no. 9464, pp. 1073-1086, 2005.

[7] N. C. van de Kar, H. G. Roelofs, H. L. Muytjens et al., "Verocytotoxin-producing Escherichia coli infection in hemolytic uremic syndrome in part of Western Europe," European Journal of Pediatrics, vol. 155, no. 7, pp. 592-595, 1996.

[8] A. R. Constantinescu, M. Bitzan, L. S. Weiss et al., "Nonenteropathic hemolytic uremic syndrome: causes and shortterm course," American Journal of Kidney Diseases, vol. 43, no. 6, pp. 976-982, 2004.

[9] R. L. Siegler, A. T. Pavia, F. L. Hansen, R. D. Christofferson, and J. B. Cook, "Atypical hemolytic-uremic syndrome: a comparison with postdiarrheal disease," Journal of Pediatrics, vol. 128, no. 4, pp. 505-511, 1996.

[10] T. J. Neuhaus, S. Calonder, and E. P. Leumann, "Heterogeneity of atypical haemolytic uraemic syndromes," Archives of Disease in Childhood, vol. 76, no. 6, pp. 518-521, 1997.

[11] A. Gerber, H. Karch, F. Allerberger, H. M. Verweyen, and L. B. Zimmerhackl, "Clinical course and the role of shiga toxinproducing Escherichia coli infection in the hemolytic-uremic syndrome in pediatric patients, 1997-2000, in Germany and Austria: a prospective study," Journal of Infectious Diseases, vol. 186, no. 4, pp. 493-500, 2002.

[12] R. Bhimma, N. C. Rollins, H. M. Coovadia, and M. Adhikari, "Post-dysenteric hemolytic uremic syndrome in children during an epidemic of Shigella dysentery in Kwazulu/Natal," Pediatric Nephrology, vol. 11, no. 5, pp. 560-564, 1997.

[13] T. G. Boyce, D. L. Swerdlow, and P. M. Griffin, "Escherichia coli O157:H7 and the hemolytic-uremic syndrome," The New England Journal of Medicine, vol. 333, no. 6, pp. 364-368, 1995.

[14] B. P. Bell, M. Goldoft, P. M. Griffin et al., "A multistate outbreak of Escherichia coli O157:H7-associated bloody diarrhea and hemolytic uremic syndrome from hamburgers: the Washington experience," Journal of the American Medical Association, vol. 272, no. 17, pp. 1349-1353, 1994.

[15] M. C. Hogan, J. M. Gloor, J. R. Uhl, F. R. Cockerill, and D. S. Milliner, "Two cases of non-O157:H7 Escherichia coli hemolytic uremic syndrome caused by urinary tract infection," American Journal of Kidney Diseases, vol. 38, no. 4, pp. E221-E226, 2001.

[16] E. Espie, F. Grimont, P. Mariani-Kurkdjian et al., "Surveillance of hemolytic uremic syndrome in children less than 15 years of age, a system to monitor O157 and non-O157 shiga toxin-producing Escherichia coli infections in France, 19962006," Pediatric Infectious Disease Journal, vol. 27, no. 7, pp. 595-601, 2008.

[17] V. Vaillant, E. Espie, H. de Valk et al., "Undercooked ground beef and person-to-person transmission as major risk factors for sporadic hemolytic uremic syndrome related to Shigatoxin producing Escherchia coli infections in children in France," The Pediatric Infectious Disease Journal, vol. 28, no. 7, pp. 650-653, 2009.

[18] N. Banatvala, P. M. Griffin, K. D. Greene et al., “The United States national prospective hemolytic uremic syndrome study: microbiologic, serologic, clinical, and epidemiologic findings," Journal of Infectious Diseases, vol. 183, no. 7, pp. 1063-1070, 2001.

[19] J. Scheiring, S. P. Andreoli, and L. B. Zimmerhackl, “Treatment and outcome of Shiga-toxin-associated hemolytic uremic syndrome (HUS)," Pediatric Nephrology, vol. 23, no. 10, pp. 1749-1760, 2008.

[20] R. E. Peterson, T. J. Klopfenstein, R. A. Moxley et al., "Efficacy of dose regimen and observation of herd immunity from a vaccine against Escherichia coli O157:H7 for feedlot cattle," Journal of Food Protection, vol. 70, no. 11, pp. 2561-2567, 2007.

[21] D. R. Smith, R. A. Moxley, T. J. Klopfenstein, and G. E. Erickson, "A randomized longitudinal trial to test the effect of regional vaccination within a cattle feedyard on Escherichia coli O157:H7 rectal colonization, fecal shedding, and hide contamination," Foodborne Pathogens and Disease, vol. 6, no. 7, pp. 885-892, 2009.

[22] D. A. Vilte, M. Larzabal, S. Garbaccio et al., "Reduced faecal shedding of Escherichia coli O157:H7 in cattle following systemic vaccination with $\gamma$-intimin $\mathrm{C}_{280}$ and EspB proteins," Vaccine, vol. 29, no. 23, pp. 3962-3968, 2011.

[23] M. A. Psotka, F. Obata, G. L. Rolling et al., "Shiga toxin 2 targets the murine renal collecting duct epithelium," Infection and Immunity, vol. 77, no. 3, pp. 959-969, 2009.

[24] C. Zoja, S. Buelli, and M. Morigi, "Shiga toxin-associated hemolytic uremic syndrome: pathophysiology of endothelial dysfunction," Pediatric Nephrology, vol. 25, no. 11, pp. 22312240, 2010

[25] M. Morigi, M. Galbusera, E. Binda et al., "Verotoxin1-induced up-regulation of adhesive molecules renders microvascular endothelial cells thrombogenic at high shear stress," Blood, vol. 98, no. 6, pp. 1828-1835, 2001. 
[26] F. Guessous, M. Marcinkiewicz, R. Polanowska-Grabowska et al., "Shiga toxin 2 and lipopolysaccharide induce human microvascular endothelial cells to release chemokines and factors that stimulate platelet function," Infection and Immunity, vol. 73, no. 12, pp. 8306-8316, 2005.

[27] L. H. Nolasco, N. A. Turner, A. Bernardo et al., "Hemolytic uremic syndrome-associated Shiga toxins promote endothelial-cell secretion and impair ADAMTS13 cleavage of unusually large von Willebrand factor multimers," Blood, vol. 106, no. 13, pp. 4199-4209, 2005.

[28] D. Orth and R. Wurzner, "Complement in typical hemolytic uremic syndrome," Seminars in Thrombosis and Hemostasis, vol. 36, no. 6, pp. 620-624, 2010.

[29] C. A. Fuller, C. A. Pellino, M. J. Flagler, J. E. Strasser, and A. A. Weiss, "Shiga toxin subtypes display dramatic differences in potency," Infection and Immunity, vol. 79, no. 3, pp. 13291337, 2011.

[30] A. Bauwens, M. Bielaszewska, B. Kemper et al., "Differential cytotoxic actions of Shiga toxin 1 and Shiga toxin 2 on microvascular and macrovascular endothelial cells," Thrombosis and Haemostasis, vol. 105, no. 3, pp. 515-528, 2011.

[31] M. Brigotti, P. L. Tazzari, E. Ravanelli et al., "Clinical relevance of shiga toxin concentrations in the blood of patients with hemolytic uremic syndrome," Pediatric Infectious Disease Journal, vol. 30, no. 6, pp. 486-490, 2011.

[32] R. L. Siegler, T. G. Obrig, T. J. Pysher, V. L. Tesh, N. D. Denkers, and F. B. Taylor, "Response to Shiga toxin 1 and 2 in a baboon model of hemolytic uremic syndrome," Pediatric Nephrology, vol. 18, no. 2, pp. 92-96, 2003.

[33] D. E. Hoey, C. Currie, R. W. Else et al., "Expression of receptors for verotoxin 1 from Escherichia coli O157 on bovine intestinal epithelium," Journal of Medical Microbiology, vol. 51, no. 2, pp. 143-149, 2002.

[34] Z. Ergonul, F. Clayton, A. B. Fogo, and D. E. Kohan, "Shigatoxin-1 binding and receptor expression in human kidneys do not change with age," Pediatric Nephrology, vol. 18, no. 3, pp. 246-253, 2003.

[35] M. A. Karmali, M. Mascarenhas, M. Petric et al., "Age-specific frequencies of antibodies to Escherichia coli verocytotoxins (Shiga toxins) 1 and 2 among urban and rural pulations in Southern Ontario," Journal of Infectious Diseases, vol. 188, no. 11, pp. 1724-1729, 2003.

[36] R. L. Siegler, "Spectrum of extrarenal involvement in postdiarrheal hemolytic-uremic syndrome," Journal of Pediatrics, vol. 125, no. 4, pp. 511-518, 1994.

[37] M. F. Gagnadoux, R. Habib, M. C. Gubler, J. L. Bacri, and M. Broyer, "Long-term (15-25 years) outcome of childhood hemolytic-uremic syndrome," Clinical Nephrology, vol. 46, no. 1, pp. 39-41, 1996.

[38] K. Blahova, J. Janda, J. Kreisinger, E. Matejkova, and A. Sediva, "Long-term follow-up of Czech children with D+ hemolytic-uremic syndrome," Pediatric Nephrology, vol. 17, no. 6, pp. 400-403, 2002.

[39] K. J. Eriksson, S. G. Boyd, and R. C. Tasker, "Acute neurology and neurophysiology of haemolytic-uraemic syndrome," Archives of Disease in Childhood, vol. 84, no. 5, pp. 434-435, 2001.

[40] N. Cimolai, B. J. Morrison, and J. E. Carter, "Risk factors for the central nervous system manifestations of gastroenteritisassociated hemolytic-uremic syndrome," Pediatrics, vol. 90, no. 4, pp. 616-621, 1992.

[41] Y. K. Jeong, I. O. Kim, W. S. Kim, Y. S. Hwang, Y. Choi, and K. M. Yeon, "Hemolytic uremic syndrome: MR findings of
CNS complications," Pediatric Radiology, vol. 24, no. 8, pp. 585-586, 1994.

[42] A. S. de Buys Roessingh, P. de Lagausie, V. Baudoin, C. Loirat, and Y. Aigrain, "Gastrointestinal complications of postdiarrheal hemolytic uremic syndrome," European Journal of Pediatric Surgery, vol. 17, no. 5, pp. 328-334, 2007.

[43] R. S. Oakes, R. L. Siegler, M. A. McReynolds, T. Pysher, and A. T. Pavia, "Predictors of fatality in postdiarrheal hemolytic uremic syndrome," Pediatrics, vol. 117, no. 5, pp. 1656-1662, 2006.

[44] R. S. Oakes, J. K. Kirkham, R. D. Nelson, and R. L. Siegler, "Duration of oliguria and anuria as predictors of chronic renal-related sequelae in post-diarrheal hemolytic uremic syndrome," Pediatric Nephrology, vol. 23, pp. 1303-1308, 2008.

[45] A. T. Serna and E. C. Boedeker, "Pathogenesis and treatment of Shiga toxin-producing Escherichia coli infections," Current Opinion in Gastroenterology, vol. 24, no. 1, pp. 38-47, 2008.

[46] B. P. Bell, P. M. Griffin, P. Lozano, D. L. Christie, J. M. Kobayashi, and P. I. Tarr, "Predictors of hemolytic uremic syndrome in children during a large outbreak of Escherichia coli O157:H7 infections," Pediatrics, vol. 100, no. 1, p. E12, 1997.

[47] J. A. Ake, S. Jelacic, M. A. Ciol et al., "Relative nephroprotection during Escherichia coli O157:H7 infections: association with intravenous volume expansion," Pediatrics, vol. 115, no. 6, pp. e673-e680, 2005.

[48] M. Bitzan, F. Schaefer, and D. Reymond, "Treatment of typical (enteropathic) hemolytic uremic syndrome," Seminars in Thrombosis and Hemostasis, vol. 36, no. 6, pp. 594-610, 2010.

[49] H. Trachtman, A. Cnaan, E. Christen et al., "Effect of an oral Shiga toxin-binding agent on diarrhea-associated hemolytic uremic syndrome in children: a randomized controlled trial," Journal of the American Medical Association, vol. 290, no. 10, pp. 1337-1344, 2003.

[50] G. L. Mulvey, P. Marcato, P. I. Kitov, J. Sadowska, D. R. Bundle, and G. D. Armstrong, "Assessment in mice of the therapeutic potential of tailored, multivalent Shiga toxin carbohydrate ligands," Journal of Infectious Diseases, vol. 187, no. 4, pp. 640-649, 2003.

[51] K. Nishikawa, M. Watanabe, E. Kita et al., "A multivalent peptide library approach identifies a novel Shiga toxin inhibitor that induces aberrant cellular transport of the toxin.," FASEB Journal, vol. 20, no. 14, pp. 2597-2599, 2006.

[52] M. A. Karmali, "Prospects for preventing serious systemic toxemic complications of Shiga toxin-producing Escherichia coli infections using Shiga toxin receptor analogues," Journal of Infectious Diseases, vol. 189, no. 3, pp. 355-359, 2004.

[53] S. Yamagami, M. Motoki, T. Kimura et al., "Efficacy of postinfection treatment with anti-Shiga toxin (Stx) 2 humanized monoclonal antibody TMA-15 in mice lethally challenged with Stx-producing Escherichia coli," Journal of Infectious Diseases, vol. 184, no. 6, pp. 738-742, 2001.

[54] T. C. Dowling, P. A. Chavaillaz, D. G. Young et al., "Phase 1 safety and pharmacokinetic study of chimeric murinehuman monoclonal antibody c alpha Stx2 administered intravenously to healthy adult volunteers," Antimicrobial Agents and Chemotherapy, vol. 49, no. 5, pp. 1808-1812, 2005.

[55] A. L. Lapeyraque, M. Malina, V. Fremeaux-Bacchi et al., "Complement blockade in severeShiga-toxin-associated HUS," The New England Journal of Medicine. In press.

[56] M. Noris and G. Remuzzi, "Genetic abnormalities of complement regulators in hemolytic uremic syndrome: how do 
they affect patient management?" Nature Clinical Practice Nephrology, vol. 1, no. 1, pp. 2-3, 2005.

[57] D. Kavanagh and T. Goodship, "Genetics and complement in atypical HUS," Pediatric Nephrology, vol. 25, no. 12, pp. 2431-2442, 2010.

[58] J. Caprioli, M. Noris, S. Brioschi et al., "Genetics of HUS: the impact of MCP, CFH, and IF mutations on clinical presentation, response to treatment, and outcome," Blood, vol. 108, no. 4, pp. 1267-1279, 2006.

[59] A. L. Sellier-Leclerc, V. Fremeaux-Bacchi, M. A. DragonDurey et al., "Differential impact of complement mutations on clinical characteristics in atypical hemolytic uremic syndrome," Journal of the American Society of Nephrology, vol. 18, no. 8, pp. 2392-2400, 2007.

[60] V. Fremeaux-Bacchi, E. J. Kemp, J. A. Goodship et al., "The development of atypical haemolytic-uraemic syndrome is influenced by susceptibility factors in factor $\mathrm{H}$ and membrane cofactor protein: evidence from two independent cohorts," Journal of Medical Genetics, vol. 42, no. 11, pp. 852856, 2005.

[61] P. Warwicker, T. H. Goodship, R. L. Donne et al., "Genetic studies into inherited and sporadic hemolytic uremic syndrome," Kidney International, vol. 53, no. 4, pp. 836-844, 1998.

[62] J. Caprioli, P. Bettinaglio, P. F. Zipfel et al., "The molecular basis of familial hemolytic uremic syndrome: mutation analysis of factor $\mathrm{H}$ gene reveals a hot spot in short consensus repeat 20," Journal of the American Society of Nephrology, vol. 12, no. 2, pp. 297-307, 2001.

[63] M. A. Dragon-Durey, V. Fremeaux-Bacchi, C. Loirat et al., "Heterozygous and homozygous factor $\mathrm{H}$ deficiencies associated with hemolytic uremic syndrome or membranoproliferative glomerulonephritis: report and genetic analysis of 16 cases," Journal of the American Society of Nephrology, vol. 15, no. 3, pp. 787-795, 2004.

[64] N. Rougier, M. D. Kazatchkine, J. P. Rougier et al., "Human complement factor $\mathrm{H}$ deficiency associated with hemolytic uremic syndrome," Journal of the American Society of Nephrology, vol. 9, no. 12, pp. 2318-2326, 1998.

[65] P. Warwicker, J. A. Goodship, and T. H. Goodship, "Factor H-US?” Nephrology Dialysis Transplantation, vol. 13, no. 8, pp. 1921-1923, 1998.

[66] S. Heinen, M. Jozsi, A. Hartmann et al., "Hemolytic uremic syndrome: a factor $\mathrm{H}$ mutation (E1172Stop) causes defective complement control at the surface of endothelial cells," Journal of the American Society of Nephrology, vol. 18, no. 2, pp. 506-514, 2007.

[67] T. S. Jokiranta, V. P. Jaakola, M. J. Lehtinen, M. Parepalo, S. Meri, and A. Goldman, "Structure of complement factor $\mathrm{H}$ carboxyl-terminus reveals molecular basis of atypical haemolytic uremic syndrome," EMBO Journal, vol. 25, no. 8, pp. 1784-1794, 2006.

[68] D. Landau, H. Shalev, G. Levy-Finer, A. Polonsky, Y. Segev, and L. Katchko, "Familial hemolytic uremic syndrome associated with complement factor H deficiency," Journal of Pediatrics, vol. 138, no. 3, pp. 412-417, 2001.

[69] M. Ohali, H. Shalev, M. Schlesinger et al., "Hypocomplementemic autosomal recessive hemolytic uremic syndrome with decreased factor H," Pediatric Nephrology, vol. 12, no. 8, pp. 619-624, 1998.

[70] A. L. Stahl, F. Vaziri-Sani, S. Heinen et al., "Factor H dysfunction in patients with atypical hemolytic uremic syndrome contributes to complement deposition on platelets and their activation," Blood, vol. 111, no. 11, pp. 5307-5315, 2008.

[71] F. Vaziri-Sani, L. Holmberg, A. G. Sjoholm et al., "Phenotypic expression of factor $\mathrm{H}$ mutations in patients with atypical hemolytic uremic syndrome," Kidney International, vol. 69, no. 6, pp. 981-988, 2006.

[72] J. Zuber, M. Le Quintrec, R. Sberro-Soussan, C. Loirat, V. Fremeaux-Bacchi, and C. Legendre, "New insights into postrenal transplant hemolytic uremic syndrome," Nature Reviews Nephrology, vol. 7, no. 1, pp. 23-35, 2011.

[73] C. Loirat and P. Niaudet, "The risk of recurrence of hemolytic uremic syndrome after renal transplantation in children," Pediatric Nephrology, vol. 18, no. 11, pp. 1095-1101, 2003.

[74] L. Couzi, C. Contin-Bordes, F. Marliot et al., "Inherited deficiency of membrane cofactor protein expression and varying manifestations of recurrent atypical hemolytic uremic syndrome in a sibling pair," American Journal of Kidney Diseases, vol. 52, no. 2, pp. e5-e9, 2008.

[75] C. J. Fang, V. Fremeaux-Bacchi, M. K. Liszewski et al., "Membrane cofactor protein mutations in atypical hemolytic uremic syndrome (aHUS), fatal Stx-HUS, C3 glomerulonephritis, and the HELLP syndrome," Blood, vol. 111, no. 2, pp. 624-632, 2008.

[76] V. Fremeaux-Bacchi, E. A. Moulton, D. Kavanagh et al., "Genetic and functional analyses of membrane cofactor protein (CD46) mutations in atypical hemolytic uremic syndrome," Journal of the American Society of Nephrology, vol. 17, no. 7, pp. 2017-2025, 2006.

[77] D. Kavanagh, E. J. Kemp, E. Mayland et al., "Mutations in complement factor I predispose to development of atypical hemolytic uremic syndrome," Journal of the American Society of Nephrology, vol. 16, no. 7, pp. 2150-2155, 2005.

[78] D. Kavanagh, A. Richards, M. Noris et al., "Characterization of mutations in complement factor I (CFI) associated with hemolytic uremic syndrome," Molecular Immunology, vol. 45, no. 1, pp. 95-105, 2008.

[79] V. Fremeaux-Bacchi, M. A. Dragon-Durey, J. Blouin et al., "Complement factor I: a susceptibility gene for atypical haemolytic uraemic syndrome," Journal of Medical Genetics, vol. 41, no. 6, p. e84, 2004.

[80] F. Bienaime, M. A. Dragon-Durey, C. H. Regnier et al., "Mutations in components of complement influence the outcome of Factor I-associated atypical hemolytic uremic syndrome," Kidney International, vol. 77, no. 4, pp. 339-349, 2010.

[81] V. Fremeaux-Bacchi, E. C. Miller, M. K. Liszewski et al., "Mutations in complement C3 predispose to development of atypical hemolytic uremic syndrome," Blood, vol. 112, no. 13, pp. 4948-4952, 2008.

[82] E. Goicoechea de Jorge, C. L. Harris, J. Esparza-Gordillo et al., "Gain-of-function mutations in complement factor B are associated with atypical hemolytic uremic syndrome," Proceedings of the National Academy of Sciences of the United States of America, vol. 104, no. 1, pp. 240-245, 2007.

[83] L. T. Roumenina, M. Jablonski, C. Hue et al., "Hyperfunctional C3 convertase leads to complement deposition on endothelial cells and contributes to atypical hemolytic uremic syndrome," Blood, vol. 114, no. 13, pp. 2837-2845, 2009.

[84] M. Delvaeye, M. Noris, A. De Vriese et al., "Thrombomodulin mutations in atypical hemolytic-uremic syndrome," The New England Journal of Medicine, vol. 361, no. 4, pp. 345-357, 2009. 
[85] M. A. Dragon-Durey, C. Loirat, S. Cloarec et al., "Anti-factor $\mathrm{H}$ autoantibodies associated with atypical hemolytic uremic syndrome," Journal of the American Society of Nephrology, vol. 16, no. 2, pp. 555-563, 2005.

[86] M. Jozsi, S. Strobel, H. M. Dahse et al., "Anti-factor H autoantibodies block C-terminal recognition function of factor $\mathrm{H}$ in hemolytic uremic syndrome," Blood, vol. 110, no. 5, pp. 1516-1518, 2007.

[87] M. A. Dragon-Durey, C. Blanc, F. Marliot et al., "The high frequency of complement factor $\mathrm{H}$ related CFHR1 gene deletion is restricted to specific subgroups of patients with atypical haemolytic uraemic syndrome," Journal of Medical Genetics, vol. 46, no. 7, pp. 447-450, 2009.

[88] C. Abarrategui-Garrido, R. Martinez-Barricarte, M. LopezTrascasa, S. R. de Cordoba, and P. Sanchez-Corral, "Characterization of complement factor H-related (CFHR) proteins in plasma reveals novel genetic variations of CFHR1 associated with atypical hemolytic uremic syndrome," Blood, vol. 114, no. 19, pp. 4261-4271, 2009.

[89] M. Jozsi, C. Licht, S. Strobel et al., "Factor H autoantibodies in atypical hemolytic uremic syndrome correlate with CFHR1/CFHR3 deficiency," Blood, vol. 111, no. 3, pp. 1512$1514,2008$.

[90] M. A. Dragon-Durey, S. K. Sethi, A. Bagga et al., "Clinical features of anti-factor $\mathrm{H}$ autoantibody-associated hemolytic uremic syndrome," Journal of the American Society of Nephrology, vol. 21, no. 12, pp. 2180-2187, 2010.

[91] O. Boyer, E. Balzamo, M. Charbit et al., "Pulse cyclophosphamide therapy and clinical remission in atypical hemolytic uremic syndrome with anti-complement factor $\mathrm{H}$ autoantibodies," American Journal of Kidney Diseases, vol. 55, no. 5, pp. 923-927, 2010.

[92] A. M. Waters, I. Pappworth, K. Marchbank et al., "Successful renal transplantation in factor $\mathrm{H}$ autoantibody associated HUS with CFHR1 and 3 deficiency and CFH variant G2850T: case report," American Journal of Transplantation, vol. 10, no. 1, pp. 168-172, 2010.

[93] J. C. Davin, L. Strain, and T. H. Goodship, "Plasma therapy in atypical haemolytic uremic syndrome: lessons from a family with a factor H mutation," Pediatric Nephrology, vol. 23, no. 9, pp. 1517-1521, 2008.

[94] V. Chatelet, T. Lobbedez, V. Fremeaux-Bacchi, M. Ficheux, J. P. Ryckelynck, and B. Hurault de Ligny, "Eculizumab: safety and efficacy after 17 months of treatment in a renal transplant patient with recurrent atypical hemolytic-uremic syndrome: case report," Transplantation Proceedings, vol. 42, no. 10, pp. 4353-4355, 2010.

[95] J. Nurnberger, T. Philipp, O. Witzke et al., "Eculizumab for atypical hemolytic-uremic syndrome," The New England Journal of Medicine, vol. 360, pp. 542-544, 2009.

[96] L. B. Zimmerhackl, J. Hofer, G. Cortina et al., "Prophylactic eculizumab after renal transplantation in atypical hemolyticuremic syndrome," The New England Journal of Medicine, vol. 362, no. 18, pp. 1746-1748, 2010.

[97] A. L. Lapeyraque, V. Fremeaux-Bacchi, and P. Robitaille, "Efficacy of eculizumab in a patient with factor-H-associated atypical hemolytic uremic syndrome," Pediatric Nephrology, vol. 26, no. 4, pp. 621-624, 2010.

[98] P. Hirt-Minkowski, S. Schaub, M. Mayr et al., "Haemolytic uraemic syndrome caused by factor $\mathrm{H}$ mutation: is single kidney transplantation under intensive plasmatherapy an option?" Nephrology Dialysis Transplantation, vol. 24, no. 11, pp. 3548-3551, 2009.
[99] D. Kavanagh, A. Richards, T. Goodship, and H. Jalanko, "Transplantation in atypical hemolytic uremic syndrome," Seminars in Thrombosis and Hemostasis, vol. 36, no. 6, pp. 653-659, 2010.

[100] J. M. Saland, P. Ruggenenti, G. Remuzzi et al., "Liverkidney transplantation to cure atypical hemolytic uremic syndrome," Journal of the American Society of Nephrology, vol. 20, no. 5, pp. 940-949, 2009.

[101] H. Jalanko, S. Peltonen, A. Koskinen et al., "Successful liverkidney transplantation in two children with aHUS caused by a mutation in complement factor $\mathrm{H}$," American Journal of Transplantation, vol. 8, no. 1, pp. 216-221, 2008.

[102] M. Furlan, "Von Willebrand factor: molecular size and functional activity," Annals of Hematology, vol. 72, no. 6, pp. 341-348, 1996.

[103] M. Furlan, R. Robles, M. Galbusera et al., "Von Willebrand factor-cleaving protease in thrombotic thrombocytopenic purpura and the hemolytic-uremic syndrome," The New England Journal of Medicine, vol. 339, no. 22, pp. 1578-1584, 1998.

[104] H. M. Tsai and E. C. Lian, "Antibodies to von Willebrand factor-cleaving protease in acute thrombotic thrombocytopenic purpura," The New England Journal of Medicine, vol. 339, no. 22, pp. 1585-1594, 1998.

[105] H. Ogier de Baulny, M. Gerard, J. M. Saudubray, and J. Zittoun, "Remethylation defects: guidelines for clinical diagnosis and treatment," European Journal of Pediatrics, vol. 157, supplement 2, pp. S77-S83, 1998.

[106] T. Kind, J. Levy, M. Lee, S. Kaicker, J. F. Nicholson, and S. A. Kane, "Cobalamin C disease presenting as hemolyticuremic syndrome in the neonatal period," Journal of Pediatric Hematology/Oncology, vol. 24, no. 4, pp. 327-329, 2002.

[107] A. P. Sharma, C. R. Greenberg, A. N. Prasad, and C. Prasad, "Hemolytic uremic syndrome (HUS) secondary to cobalamin C (cblC) disorder," Pediatric Nephrology, vol. 22, no. 12, pp. 2097-2103, 2007.

[108] J. A. Groff, M. Kozak, J. P. Boehmer, T. M. Demko, and J. R. Diamond, "Endotheliopathy: a continuum of hemolytic uremic syndrome due to mitomycin therapy," American Journal of Kidney Diseases, vol. 29, no. 2, pp. 280-284, 1997.

[109] J. B. Lesesne, N. Rothschild, B. Erickson et al., "Cancerassociated hemolytic-uremic syndrome: analysis of 85 cases from a national registry," Journal of Clinical Oncology, vol. 7, no. 6, pp. 781-789, 1989.

[110] S. Becker, G. Fusco, J. Fusco et al., "HIV-associated thrombotic microangiopathy in the era of highly active antiretroviral therapy: an observational study," Clinical Infectious Diseases, vol. 39, supplement 5, pp. S267-S275, 2004.

[111] C. Gervasoni, A. L. Ridolfo, M. Vaccarezza et al., "Thrombotic microangiopathy in patients with acquired immunodeficiency syndrome before and during the era of introduction of highly active antiretroviral therapy," Clinical Infectious Diseases, vol. 35, no. 12, pp. 1534-1540, 2002.

[112] S. Vasoo, J. Thumboo, and K. Y. Fong, "Thrombotic thrombocytopenic purpura in systemic lupus erythematosus: disease activity and the use of cytotoxic drugs," Lupus, vol. 11, no. 7, pp. 443-450, 2002.

[113] J. Zuber, M. Le Quintrec, R. Sberro-Soussan, C. Loirat, V. Fremeaux-Bacchi, and C. Legendre, "New insights into postrenal transplant hemolytic uremic syndrome," Nature Reviews Nephrology, vol. 7, no. 1, pp. 23-35, 2011. 
[114] H. M. Trimarchi, L. D. Truong, S. Brennan, J. M. Gonzalez, and W. N. Suki, "FK506-associated thrombotic microangiopathy: report of two cases and review of the literature," Transplantation, vol. 67, no. 4, pp. 539-544, 1999.

[115] J. A. Wolfe, R. L. McCann, and F. Sanfilippo, "Cyclosporineassociated microangiopathy in renal transplantation: a severe but potentially reversible form of early graft injury," Transplantation, vol. 41, no. 4, pp. 541-544, 1986. 


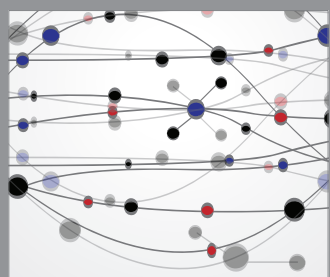

The Scientific World Journal
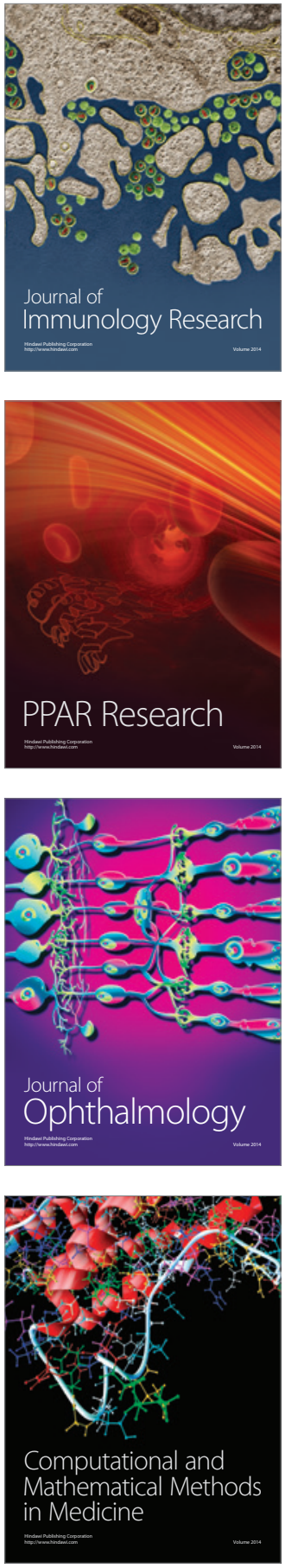

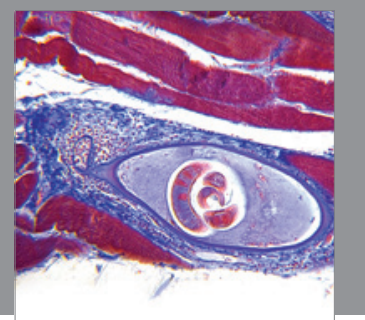

Gastroenterology

Research and Practice
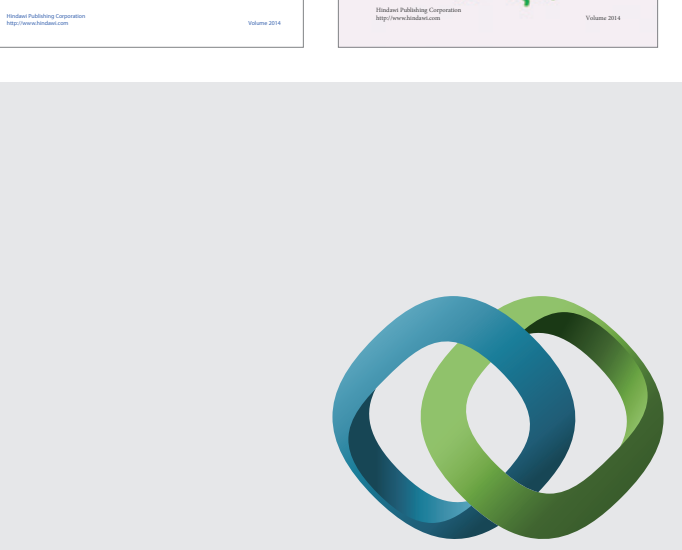

\section{Hindawi}

Submit your manuscripts at

http://www.hindawi.com
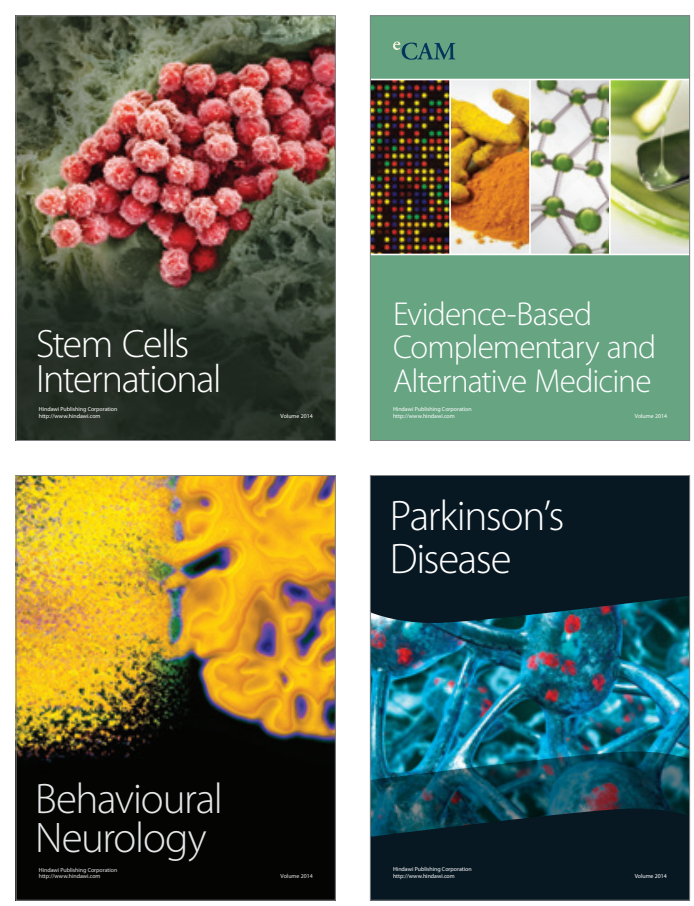

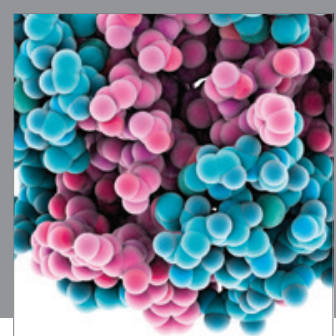

Journal of
Diabetes Research

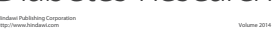

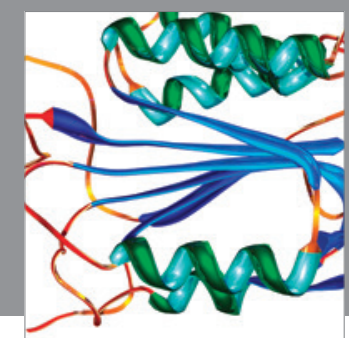

Disease Markers
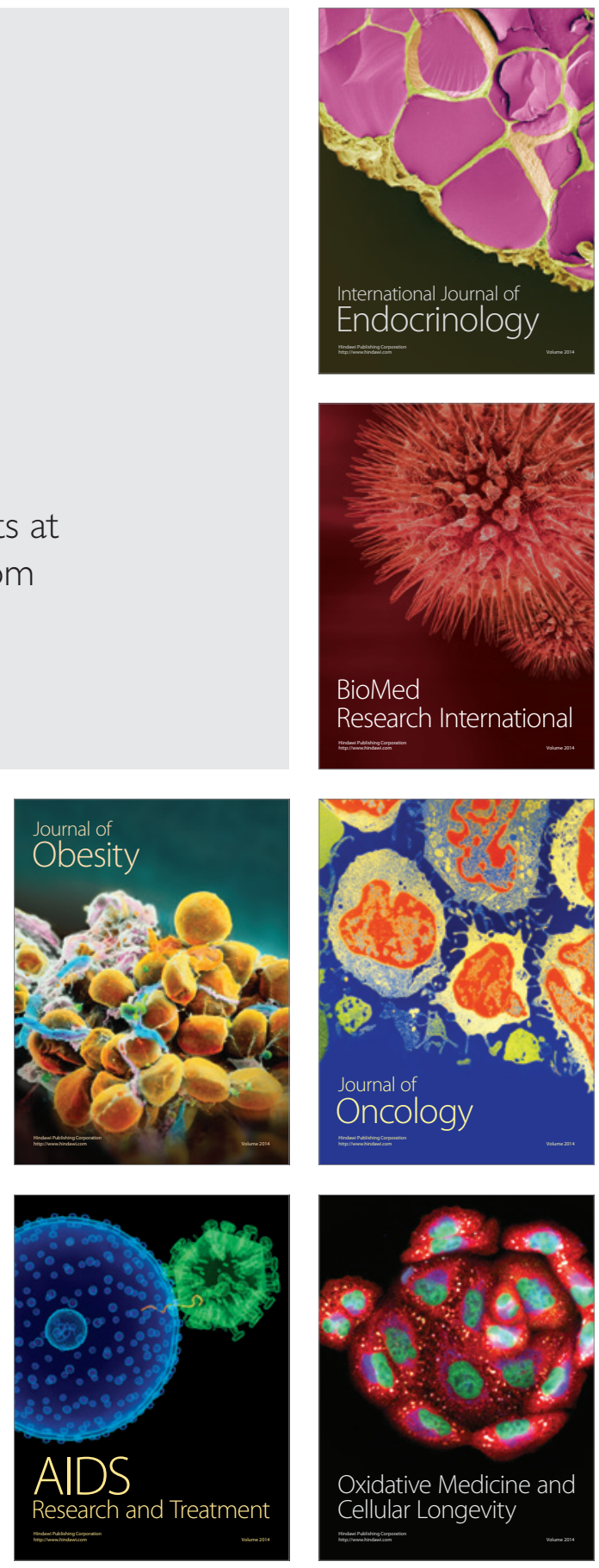